\title{
DIFFERENTIABILITY OF THE NORM IN VON NEUMANN ALGEBRAS
}

\author{
KEITH F. TAYLOR AND WEND WERNER
}

(Communicated by Palle E. T. Jorgensen)

\begin{abstract}
Smooth points in von Neumann algebras are characterized in terms of minimal projections. The theorem generalizes known results for the algebra $L^{\infty}(\Omega, \Sigma, \mu)$ and the space of bounded linear operators on a Hilbert space.
\end{abstract}

\section{INTRODUCTION}

Let $X$ be a Banach space with norm $\|\cdot\|$ and unit ball $B_{X}$. Following the generally adopted notion in Banach space theory, we call a point $x \in X$ smooth if the norm is Gâteaux differentiable at $x$, i.e., if the directional derivatives

$$
\varphi_{x}(y)=\lim _{h \rightarrow 0} \frac{\|x+h y\|-\|x\|}{h}
$$

exist, for all directions $y$. Due to the convexity of the norm function, the mapping $\varphi_{x}$ is a real continuous functional. Also $\left\|\varphi_{x}\right\|=1$ and $\varphi_{x}(x)=\|x\|$. As a matter of fact, the point $x$ is smooth if and only if there is only one continuous functional with this property. (This also holds true for complex spaces.)

If the limit in $(*)$ is uniform in $y$, i.e., if

$$
\lim _{\|y\| \rightarrow 0} \frac{\|x+y\|-\|x\|-\varphi_{x}(y)}{\|y\|}=0,
$$

then the norm is called Fréchet differentiable at $x$. Clearly, Fréchet differentiability of the norm at $x$ implies Gâteaux differentiability of the norm at $x$.

For a more detailed exposition we refer to [6].

Recently, Kittaneh and Younis [5] characterized the smooth points in $\mathfrak{B}(\mathscr{H})$, the Banach algebra of all bounded linear operators on a Hilbert space $\mathscr{H}$. On the other hand, the smooth points in $L^{\infty}(\Omega, \Sigma, \mu)$, for a measure space $(\Omega, \Sigma, \mu)$, have been known for a long time. Both $\mathfrak{B}(\mathscr{H})$ and $L^{\infty}(\Omega, \Sigma, \mu)$ are particular examples of von Neumann algebras. In the theorem below, we give characterizations of the smooth points in any von Neumann algebra, which

Received by the editors February 10, 1992.

1991 Mathematics Subject Classification. Primary 46L10, 46B20.

The first author was partially supported by an NSERC Canada Operating Grant. 
extend the previously mentioned results for $\mathfrak{B}(\mathscr{H})$ and $L^{\infty}(\Omega, \Sigma, \mu)$. Moreover, we are able to show that the norm is Fréchet differentiable at a point in a von Neumann algebra if and only if it is a smooth point. This is obvious for the case of $L^{\infty}(\Omega, \Sigma, \mu)$.

Theorem. Let $\mathfrak{M}$ be a von Neumann algebra and let $T \in \mathfrak{M}$. The following assertions are equivalent:

(a) $T$ is a smooth point of $\mathfrak{M}$.

(b) $|T|$ is a smooth point of $\mathfrak{M}$.

(c) $\|T\|$ is an isolated point in the spectrum of $|T|$ and the corresponding spectral projection is a minimal projection in $\mathfrak{M}$.

(d) There exists a minimal projection $P$ in $\mathfrak{M}$ such that

$$
\||| T \mid P\|=\|T\| \text { and }\|T \mid(I-P)\|<\|T\| .
$$

(e) There exists a minimal projection $P$ in $\mathfrak{M}$ such that

$$
\|T P\|=\|T\| \text { and }\|T(I-P)\|<\|T\| .
$$

(f) The norm in $\mathfrak{M}$ is Fréchet differentiable at $T$.

The proof of this theorem is given in $\S 3$, while $\S 2$ contains the necessary information on von Neumann algebras. In $\S 4$ the concluding remarks relate these characterizations to other work.

\section{Von NeUmanN ALgebras}

In this section, the definition and relevant properties of von Neumann algebras are listed. The books of Kadison and Ringrose [4] and Takesaki [7] provide excellent references for the rich theory of these algebras.

2.1. A $C^{*}$-algebra is a Banach algebra $\mathfrak{A}$ with involution, $T \rightarrow T^{*}$, such that $\left\|T^{*} T\right\|=\|T\|^{2}$. A von Neumann algebra is a $C^{*}$-algebra $\mathfrak{M}$ with a predual; that is, there exists a Banach space $\mathfrak{M}_{*}$ such that $\mathfrak{M}$ is isometrically isomorphic to the dual $\left(\mathfrak{M}_{*}\right)^{*}$ of $\mathfrak{M}_{*}$. It turns out that $\mathfrak{M}_{*}$ is necessarily unique up to isometric isomorphism. Of course, $\mathfrak{M}_{*}$ can be considered as a closed subspace of the dual $\mathfrak{M}^{*}$ of $\mathfrak{M}$ and the continuous linear functionals on $\mathfrak{M}$, which lie in $\mathfrak{M}_{*}$, are called normal linear functionals on $\mathfrak{M}$.

2.2. Von Neumann algebras always have an identity, denoted $I$ throughout this paper, and an order structure. For $T \in \mathfrak{M}$, let $\operatorname{sp}(T)$ denote the spectrum of $T$. If $T$ is selfadjoint $\left(T^{*}=T\right)$ and $\operatorname{sp}(T) \subseteq[0, \infty)$, then $T$ is called positive. $T$ is positive if and only if $T=S^{*} S$, for some $S \in \mathfrak{M}$, and if and only if $T=R^{2}$, for some positive $R \in \mathfrak{M}$. We write $T \geq 0$ if $T$ is positive. For any $T \in \mathfrak{M}$, let $|T|=\left(T^{*} T\right)^{1 / 2}$, using continuous functional calculus. There exists a unique partial isometry $V$ in $\mathfrak{M}$ such that $T=V|T|$ and $V V^{*}$ is the projection in $\mathfrak{M}$ with the property that $\left(V V^{*}\right) T=T$ and if $P$ is a projection in $\mathfrak{M}$ such that $P T=T$ then $V V^{*} \leq P$. Moreover, $V^{*} T=|T|$.

2.3. Any von Neumann algebra $\mathfrak{M}$ can be faithfully represented as an algebra of bounded operators on some Hilbert space. More precisely, there exists a Hilbert space $\mathscr{H}$ and an isometric ${ }^{*}$-isomorphism of $\mathfrak{M}$ onto a ${ }^{*}$-subalgebra of $\mathfrak{B}(\mathscr{H})$, which is closed in the weak operator topology. One can also ensure that the identity $I$ of $\mathfrak{M}$ is mapped to the identity in $\mathfrak{B}(\mathscr{H})$. Then the terms 
selfadjoint, positive, projection, and partial isometry appearing in 2.2 have their usual meaning as operators on $\mathscr{H}$.

2.4. The Spectral Theorem. If $S$ is a selfadjoint element of $\mathfrak{M}$, then $\operatorname{sp}(S)$ is real and contained in the interval $[-\|S\|,\|S\|]$. Moreover, there exists a projection-valued measure $B \mapsto E(B)$ from the Borel subsets of $[-\|S\|,\|S\|]$ into $P(\mathfrak{M})$, the lattice of projections in $\mathfrak{M}$, such that $S=\int_{-\|S\|}^{\|S\|} \lambda d E(\lambda)$. If $S \geq 0$, then $E$ is supported on $[0,\|S\|]$. If $\lambda_{0}$ is an isolated point in $\operatorname{sp}(S)$, then $P=E\left(\left\{\lambda_{0}\right\}\right)$ is a nonzero projection in $\mathfrak{M}$ such that $S P=P S=\lambda_{0} P$.

2.5. For $\varphi \in \mathfrak{M}^{*}$, define $\varphi^{*} \in \mathfrak{M}^{*}$ by $\varphi^{*}(S)=\overline{\varphi\left(S^{*}\right)}$ for all $S \in \mathfrak{M}$. Then $\left\|\varphi^{*}\right\|=\|\varphi\|$ for all $\varphi \in \mathfrak{M}^{*}$. If $T \geq 0$ implies $\varphi(T) \geq 0$ for $T \in M$, then $\varphi$ is called positive and we write $\varphi \geq 0$. If $\varphi \geq 0$, then $\varphi^{*}=\varphi$.

2.6. There are natural left and right actions of $\mathfrak{M}$ on $\mathfrak{M}^{*}$ which make $\mathfrak{M}^{*}$ into a 2-sided Banach $\mathfrak{M}$-module. For $A \in \mathfrak{M}$ and $\varphi \in \mathfrak{M}^{*}$ define $A \varphi$ and $\varphi A$ in $\mathfrak{M}^{*}$ by $(A \varphi)(B)=\varphi(B A)$ and $(\varphi A)(B)=\varphi(A B)$ for all $B \in \mathfrak{M}$. Then

(i) $\|A \varphi\| \leq\|A\|\|\varphi\|$ and $\|\varphi A\| \leq\|\varphi\|\|A\|$;

(ii) $(A \varphi)^{*}=\varphi^{*} A^{*}$;

(iii) $\varphi \in \mathfrak{M}_{*}$ implies $A \varphi, \varphi A \in \mathfrak{M}_{*}$.

2.7. The next lemma generalizes [7, Lemma III.4.1].

Lemma. Let $\left(\psi_{n}\right)$ be a sequence in $\mathfrak{M}^{*}$, with $\left\|\psi_{n}\right\| \leq 1$, for all $n$. Suppose $P$ and $Q$ are projections in $\mathfrak{M}$ such that $\left\|P \psi_{n} Q\right\| \rightarrow 1$ as $n \rightarrow \infty$. Then $\left\|P \psi_{n} Q-\psi_{n}\right\| \rightarrow 0$ as $n \rightarrow \infty$.

Proof. Since $\left\|P \psi_{n} Q\right\| \leq\left\|P \psi_{n}\right\| \leq 1$, we have $\left\|P \psi_{n}\right\| \rightarrow 1$. If $\left\|P \psi_{n}-\psi_{n}\right\| \nrightarrow 0$, then there exists a $\delta>0$ and $n \in \mathbb{N}$ such that $\left\|P \psi_{n}-\psi_{n}\right\|>\delta$ and $\left\|P \psi_{n}\right\|>$ $\left(1+\delta^{2}\right)^{1 / 2}-\delta^{2}$.

Fix $A, B \in \mathfrak{M},\|A\|,\|B\| \leq 1$ such that $\left(P \psi_{n}\right)(A)>\left(1+\delta^{2}\right)^{1 / 2}-\delta^{2}$ and $\left((I-P) \psi_{n}\right)(B)>\delta$. Since $\|S\|=\left\|S S^{*}\right\|^{1 / 2}$, for all $S \in \mathfrak{M}$, we have

$$
\|A P+\delta B(I-P)\|=\left\|A P A^{*}+\delta^{2} B(I-P) B^{*}\right\|^{1 / 2} \leq\left(1+\delta^{2}\right)^{1 / 2} .
$$

However, $\psi_{n}(A P+\delta B(I-P))=\left(P \psi_{n}\right)(A)+\delta\left((I-P) \psi_{n}\right)(B)>\left(1+\delta^{2}\right)^{1 / 2}$. This contradicts $\left\|\psi_{n}\right\| \leq 1$.

Analogously, or applying the previous paragraph to $Q \psi_{n}^{*}$, we have $\| \psi_{n} Q-$ $\psi_{n} \| \rightarrow 0$ and also $\left\|P \psi_{n} Q-\psi_{n} Q\right\| \rightarrow 0$ as $n \rightarrow \infty$. Then

$$
\left\|P \psi_{n} Q-\psi_{n}\right\| \leq\left\|P \psi_{n} Q-\psi_{n} Q\right\|+\left\|\psi_{n} Q-\psi_{n}\right\| \rightarrow 0 \text { as } n \rightarrow \infty \text {. }
$$

\section{Proof of THE THEOREM}

As usual, we assume, without loss of generality, that $\|T\|=1$ for the duration of the proof. The scheme of the proof is to show $(a) \Rightarrow(c) \Rightarrow(d) \Rightarrow(e) \Rightarrow$ (f) $\Rightarrow(a)$. Then (b) is obviously equivalent to the other conditions by replacing $T$ with $|T|$. Let $T=V|T|$ be the polar decomposition of $T$, as in 2.2 .

(a) $\Rightarrow$ (c) Assume that $T$ is a smooth point of $\mathfrak{M}$. Let $E$ be the projectionvalued measure from $[0,1]$ into $P(\mathfrak{M})$ such that

$$
|T|=\int_{0}^{1} \lambda d E(\lambda) \quad \text { as in } 2.4 \text {. }
$$


Let $\mathfrak{M}(|T|)$ denote the von Neumann subalgebra of $\mathfrak{M}$ generated by $\{|T|, I\}$. Then $\mathfrak{M}(|T|)$ is a commutative von Neumann algebra and $E(B) \in \mathfrak{M}(|T|)$ for any Borel subset $B$ of $[0,1]$. Let $E_{n}=E\left[1-\frac{1}{n}, 1-\frac{1}{n+1}\right)$ for $n=1,2, \ldots$.

Note first that 1 is a point in $\operatorname{sp}(|T|)$. If 1 is not isolated in $\operatorname{sp}(|T|)$, then $J=\left\{n \in \mathbb{N}: E_{n} \neq 0\right\}$ is an infinite set, say $J=\left\{n_{1}, n_{2}, n_{3}, \ldots\right\}$. Let $F_{1}=$ $\sum_{k=1}^{\infty} E_{n_{2 k-1}}$ and $F_{2}=\sum_{k=1}^{\infty} E_{n_{2 k}}$. Then $F_{1}$ and $F_{2}$ are projections in $\mathfrak{M}(|T|)$ satisfying $F_{1} F_{2}=0$. Now $|T| E_{n} \geq\left(1-\frac{1}{n}\right) E_{n}$; so $\left\||T| E_{n}\right\| \geq 1-\frac{1}{n}$, for each $n \in J$. Thus $\left\||T| F_{1}\right\|=1$ and $\left\||| T \mid F_{2}\right\|=1$. Since $V^{*} T=|T|$, we have $1 \geq\left\|T F_{1}\right\| \geq\left\|V^{*} T F_{1}\right\|=\|\| T \mid F_{1} \|=1$. Likewise, $\left\|T F_{2}\right\|=1$. Now let $\varphi_{1}, \varphi_{2} \in \mathfrak{M}^{*}$ be such that $\left\|\varphi_{1}\right\|=\left\|\varphi_{2}\right\|=1$ and $\varphi_{1}\left(T F_{1}\right)=\varphi_{2}\left(T F_{2}\right)=1$. Let $\rho_{1}, \rho_{2} \in \mathfrak{M}^{*}$ be defined by $\rho_{1}(S)=\varphi_{1}\left(S F_{1}\right)$ and $\rho_{2}(S)=\varphi_{2}\left(S F_{2}\right)$ for all $S \in \mathfrak{M}$. Then $\left\|\rho_{1}\right\|=\left\|\rho_{2}\right\|=1$ and $\rho_{1}(T)=\rho_{2}(T)=1$; however, $\rho_{1}\left(T F_{2}\right)=\varphi_{1}\left(T F_{2} F_{1}\right)=0 \neq \varphi_{2}\left(T F_{2} F_{2}\right)=\rho_{2}\left(T F_{2}\right)=1$. Thus $\rho_{1} \neq \rho_{2}$ and this contradicts the fact that $T$ is a smooth point in $\mathfrak{M}$.

Therefore, 1 is an isolated point in $\operatorname{sp}(|T|)$. This implies that $E(\{1\})$ is a nonzero projection in $\mathfrak{M}$. If there exists an $F_{1} \in P(\mathfrak{M})$ with $0 \neq F_{1} \leq E(\{1\})$ and $F_{1} \neq E(\{1\})$, then let $F_{2}=E(\{1\})-F_{1}$. As above, $\left\|T F_{1}\right\|=\left\|T F_{2}\right\|=1$ and one sees that $T$ cannot be a smooth point. Thus $E(\{1\})$ must be a minimal projection in $\mathfrak{M}$.

(c) $\Rightarrow$ (d) is obvious.

(d) $\Rightarrow$ (e) If $\||T| P\|=1$ and $\||| T \mid(I-P)\|<1$, then, with the same $P$, $1 \geq\|T P\| \geq\left\|V^{*} T P\right\|=\|T \mid P\|=1$ and $\|T(I-P)\|=\|V|T|(I-P)\| \leq$ $\||| T \mid(I-P)\|<1$.

(e) $\Rightarrow$ (f) As before, let $T=V|T|$ be the polar decomposition of $T$ and let $\int_{0}^{1} \lambda d E(\lambda)$ be the spectral representation of $|T|$. Let us first show that $P=E(\{1\})$ and $I-P=E([0, h])$ for some $h<1$ :

To this end, denote by $c(P)$ the central cover of $P$, i.e., the smallest central projection in $\mathfrak{M}$ dominating $P$. Then $c(P) \mathfrak{M}$ has trivial center, $P=c(P) P$ is minimal in $c(P) \mathfrak{M}$, and hence, $c(P) \mathfrak{M}$ is a type $I$ factor. This implies $c(P) \mathfrak{M}=\mathfrak{B}\left(\mathscr{H}_{0}\right)$ for some Hilbert space $\mathscr{H}_{0}$ [7, Corollary V.1.28]. It follows that $T P$ is a partial isometry and $P T^{*} T P=P$. This yields $P\left(I-|T|^{2}\right) P=0$, and since $I-|T|^{2} \geq 0$, we may conclude that $P\left(I-|T|^{2}\right)^{1 / 2}=\left(I-|T|^{2}\right)^{1 / 2} P=0$; but then $P|T|^{2}=|T|^{2} P=P$, and the above claim is now obvious.

To show (f), represent $\mathfrak{M}$ on $\mathscr{H}=\mathscr{H}_{0} \oplus \mathscr{H}_{1}$ such that $c(P) \mathfrak{M}=\mathfrak{B}_{\left(\mathscr{H}_{0}\right) \text { and }}$ $(I-c(P)) \mathfrak{M} \subseteq \mathfrak{B}\left(\mathscr{H}_{1}\right)$ for some Hilbert space $\mathscr{H}_{1}$. Define support functionals in $\mathfrak{M}_{*}$ of $|T|$ and $T$ as follows: Let $\xi$ be a unit vector in $P(\mathscr{H})$ and let

$$
\varphi(S)=\langle S \xi, \xi\rangle \quad \text { and } \quad \varphi^{\prime}(S)=\langle S \xi, V \xi\rangle \quad \text { for all } S \in \mathfrak{M} \text {. }
$$

Then, $\varphi, \varphi^{\prime} \in \mathfrak{M}_{*},\|\varphi\|=\left\|\varphi^{\prime}\right\|=1, \varphi(|T|)=1$, and

$$
\varphi^{\prime}(T)=\langle T \xi, V \xi\rangle=\left\langle V^{*} T \xi, \xi\right\rangle=\langle|T| \xi, \xi\rangle=1 .
$$

Note that $P \varphi=\varphi$ and $P \varphi^{\prime}=\varphi^{\prime}$.

By a classical result of Šmulyan (see, e.g., [6, 5.11 Proposition]), the norm is Fréchet differentiable at $T$ if and only if $\varphi^{\prime}$ is a strongly exposed point in $\mathfrak{M}^{*}$; that is, it suffices to show that if $\left(\varphi_{n}^{\prime}\right)$ is a sequence in the unit ball of $\mathfrak{M}^{*}$ such that $\varphi_{n}^{\prime}(T) \rightarrow 1$ then $\left\|\varphi_{n}^{\prime}-\varphi^{\prime}\right\| \rightarrow 0$. Let $\left(\varphi_{n}^{\prime}\right)$ be such a sequence and, for each $n$, let $\varphi_{n}=\varphi_{n}^{\prime} V$. Then $\varphi_{n}(|T|)=\varphi_{n}^{\prime}(V|T|)=\varphi_{n}^{\prime}(T) \rightarrow 1$ and, of course $\left\|\varphi_{n}\right\| \leq 1$ for all $n$. 
Claim. $\varphi_{n}(P) \rightarrow 1$. To see this we use the functional calculus (see [4, 5.2.9 Theorem]). There is a regular Borel measure $\mu$ on $\operatorname{sp}(|T|)$ and the map $f \rightarrow$ $f(|T|)=\int_{0}^{1} f(\lambda) d E(\lambda)$ is a *-isomorphism of $L^{\infty}(\operatorname{sp}(|T|), \mu)$ with $\mathfrak{M}(|T|)$, the von Neumann subalgebra of $\mathfrak{M}$ generated by $|T|$ and $I$. For each $n$, let $\psi_{n}$ represent $\varphi_{\left.n\right|_{\mathfrak{m}(|T|)}}$ as a linear functional on $L^{\infty}(\operatorname{sp}(|T|)$. Then $P$ in $\mathfrak{M}(|T|)$ corresponds to $\chi_{\{1\}}$, the characteristic function of the atom $\{1\}$ in $\operatorname{sp}(|T|)$. Also $|T|$ corresponds to the identity function $l$, where $l(\lambda)=\lambda$ for all $\lambda \in \operatorname{sp}(|T|)$. Thus, $\psi_{n}(l) \rightarrow 1$. For a continuous linear functional $\psi$ on $L^{\infty}(\operatorname{sp}(|T|), \mu)$ and some measurable set $A \subseteq \operatorname{sp}(|T|)$ define $\psi \chi_{A}$ on $L^{\infty}(\operatorname{sp}(|T|), \mu)$ by $\psi \chi_{A}(f)=\psi\left(\chi_{A} f\right)$ for all $f \in L^{\infty}(\operatorname{sp}(|T|), \mu)$. If $0<h<1$ is as above, it is easily seen that $\|\psi\|=\left\|\psi \chi_{[0, h]}\right\|+\left|\psi\left(\chi_{\{1\}}\right)\right|$. Since $\psi_{n}(l) \leq$ $h\left\|\psi_{n} \chi_{[0, h]}\right\|+\left|\psi_{n}\left(\chi_{\{1\}}\right)\right|, \psi_{n}(l) \rightarrow 1$, and $\left\|\psi_{n} \chi_{[0, h]}\right\|+\left|\psi_{n}\left(\chi_{\{1\}}\right)\right|=\left\|\psi_{n}\right\| \leq 1$, we must have $\left\|\psi_{n} \chi_{[0, h]}\right\| \rightarrow 0$ and $\left|\psi_{n}\left(\chi_{\{1\}}\right)\right| \rightarrow 1$. Since $\psi_{n}(l)=\psi_{n} \chi_{[0, h]}(l)+$ $\psi_{n}\left(\chi_{\{1\}}\right)$, it follows that $\psi_{n}\left(\chi_{\{1\}}\right) \rightarrow 1$; but this corresponds to $\varphi_{n}(P) \rightarrow 1$, and we have proven the claim.

Now, the projection $V^{*} V$ dominates $P$, so $Q=V P V^{*}$ is a projection in $\mathfrak{M}$ and

$$
P \varphi_{n}^{\prime} Q(V)=\varphi_{n}^{\prime}(Q V P)=\varphi_{n}^{\prime}\left(V P V^{*} V P\right)=\varphi_{n}^{\prime}(V P)=\varphi_{n}(P) \rightarrow 1 .
$$

Thus $\left\|P \varphi_{n}^{\prime} Q\right\| \rightarrow 1$. Lemma 2.7 implies that $\left\|P \varphi_{n}^{\prime} Q-\varphi_{n}^{\prime}\right\| \rightarrow 0$. Clearly, $P$ and $Q$ both belong to $c(P) \mathfrak{M}$ and hence, on $\mathscr{H}$ as chosen above, they correspond to rank one projections. Therefore, $Q \mathfrak{M} P=\mathbb{C}(V P)$. Also $P \varphi^{\prime} Q=\varphi^{\prime}$, and thus, for any $\psi \in \mathfrak{M}^{*}, P \psi Q=\alpha \varphi^{\prime}$, where $\alpha \in \mathbb{C}$ (in fact, $\alpha=\psi(V P)$ ). In particular, $P \varphi_{n}^{\prime} Q=\alpha_{n} \varphi^{\prime}$, where $\alpha_{n}=\varphi_{n}^{\prime}(V P) \rightarrow 1$. Now we can show that $\varphi_{n}^{\prime} \rightarrow \varphi^{\prime}$ in norm. For

$$
\begin{aligned}
\left\|\varphi^{\prime}-\varphi_{n}^{\prime}\right\| & \leq\left\|\varphi^{\prime}-P \varphi_{n}^{\prime} Q\right\|+\left\|P \varphi_{n}^{\prime} Q-\varphi_{n}^{\prime}\right\| \\
& =\left|1-\alpha_{n}\right|+\left\|P \varphi_{n}^{\prime} Q-\varphi_{n}^{\prime}\right\| \rightarrow 0 \text { as } n \rightarrow \infty .
\end{aligned}
$$

(f) $\Rightarrow$ (a) is immediate and this completes the proof of the theorem.

\section{CONCLUding REMARKS}

4.1. If $\mathfrak{M}$ is a continuous von Neumann algebra, such as Type II or Type III factor, then $\mathfrak{M}$ has no smooth points since it has no minimal projections.

4.2. If $\mathfrak{M}=L^{\infty}(\Omega, \Sigma, \mu)$, then we recover the well-known result that the norm in $L^{\infty}(\Omega, \Sigma, \mu)$ is Gâteaux differentiable at $f$ if and only if it is Fréchet differentiable at $f$, which holds if and only if there is an atom $E$ in $\Sigma$ such that $|f(E)|=\|f\|$ and $\left\|f \chi_{\Omega \backslash E}\right\|<\|f\|$.

4.3. If $\mathfrak{M}=\mathfrak{B}(\mathscr{H})$, then our theorem states that for $T \in \mathfrak{B}(\mathscr{H})$ the following are equivalent:

(1) $T$ is a smooth point.

(2) There exists a rank 1 projection $P$ on $\mathscr{H}$ such that $\|T P\|=\|T\|$ and $\|T(I-P)\|<\|T\|$.

(3) $T$ is a point of Fréchet differentiability of the norm.

Similar to the first part of (e) $\Rightarrow$ (f) one can derive Theorem 2 of [5] from the equivalence of (1) and (2). It seems to be much easier to check if (2) holds than the condition on the essential norm of $T$ in [5]. 
From $(2) \Leftrightarrow(3)$, we recover the $\mathfrak{B}(\mathscr{H})$ version of the criterion for Fréchet differentiability of the norm given by Heinrich in [3]. (Heinrich gives criteria for $\mathfrak{B}(E, F)$ with $E$ and $F$ arbitrary Banach spaces.) It does not seem to have been observed before that (1) and (3) are equivalent in $\mathfrak{B}(\mathscr{H})$.

4.4. Recently, the facial structure of the unit ball in a von Neumann algebra $\mathfrak{M}$ was completely described by Edwards and Rüttimann [2]. (Actually, they deal with the more general case of $J B W^{*}$-triples. See also Akemann and Pedersen [1], where the von Neumann algebra versions of the results of [2] are also presented and extended to $C^{*}$-algebras.) Let $B_{\mathfrak{M}}$ denote the unit ball of $\mathfrak{M}$. Any weak*-closed face $F$ of $B_{\mathfrak{M}}$ then has the form

$$
F_{U}=U+\left(I-U U^{*}\right) B_{\mathfrak{M}}\left(I-U^{*} U\right)=\left\{T \in B_{\mathfrak{M}} \mid U U^{*}=T U^{*}\right\}
$$

for some partial isometry $U$ in $\mathfrak{M}$.

By our theorem, $T$ is smooth if and only if there is a partial isometry $U$ such that $U^{*} U$ is minimal, $T U^{*}=U U^{*}$, and $\|T-U\|<1$. In fact, whenever $T$ is smooth and $P$ is the minimal projection as in (c), we may take $U=V P$, where $T=V|T|$ is the polar decomposition of $T$. In light of (e) this condition implies easily that $T$ is a smooth point.

The above shows that the smooth points of $B_{\mathfrak{M}}$ precisely find themselves in the interior of the faces $F_{U}$ where $U$ has the property that $U^{*} U$ is a minimal projection.

\section{ACKNOWLEDGMENT}

This research was carried out while the first author was a guest at Univ. G-H. Paderborn. He expresses his heartfelt thanks to Professor Kaniuth and his colleagues for their hospitality.

\section{REFERENCES}

1. C. A. Akemann and G. K. Pedersen, Facial structure in operator algebra theory, Proc. London Math. Soc. 64 (1992), 418-448.

2. C. M. Edwards and G. T. Rüttimann, On the facial structure of the unit balls in a $J B W^{*}$. triple and its dual, J. London Math. Soc. (2) 38 (1988), 317-332.

3. S. Heinrich, The differentiability of the norm in spaces of operators, Funct. Anal. Appl. 9 (1975), 360-362.

4. R. V. Kadison and J. R. Ringrose, Fundamentals of the theory of operator algebras. I, II, Academic Press, New York and London, 1983, 1986.

5. F. Kittaneh and R. Younis, Smooth points of certain operator spaces, Integral Equations Operator Theory 13 (1990), 849-855.

6. R. R. Phelps, Convex functions, monotone operators and differentiability, Lecture Notes in Math., vol. 1364, Springer, Berlin, Heidelberg, and New York, 1989.

7. M. Takesaki, Theory of operator algebras. I, Springer, Berlin, Heidelberg, and New York, 1979.

Department of Mathematics, University of Saskatchewan, Saskatoon, Saskatchewan, CANADA S7N 0W0

E-mail address: taylor@skmath1.usask.ca

UNiversität-Gesamthochschule-PAderboRn, FaChbereich 17, Postfach 1621, 4790 PAderborn, Germany

E-mail address: wend@uni-paderborn.de 